\title{
La représentation du travail dans les photographies publicitaires des Éditions Paul-Martial
}

The representation of the work in the advertising photographs of Editions PaulMartial

\section{Anne-Céline Callens}

\section{OpenEdition}

\section{Journals}

Édition électronique

URL : http://journals.openedition.org/itti/359

DOI : 10.4000/itti.359

Éditeur

Université de Poitiers

\section{Référence électronique}

Anne-Céline Callens, «La représentation du travail dans les photographies publicitaires des Éditions Paul-Martial », Images du travail, travail des images [En ligne], 8| 2020, mis en ligne le 01 février 2020 consulté le 14 avril 2021. URL : http://journals.openedition.org/itti/359 ; DOI : https://doi.org/10.4000/ itti.359

Ce document a été généré automatiquement le 14 avril 2021

Images du travail, travail des images 


\section{La représentation du travail dans les photographies publicitaires des Éditions Paul-Martial}

The representation of the work in the advertising photographs of Editions PaulMartial

Anne-Céline Callens

\section{NOTE DE L'AUTEUR}

L'étude du fonds Paul-Martial du musée d'art moderne et contemporain de SaintÉtienne Métropole a bénéficié des travaux en Humanités Numériques réalisés dans le cadre du programme de recherche Viva-Arts dirigé par Philippe Colantoni, maître de conférences en génie informatique, automatique et traitement du signal, et du soutien financier de Saint Étienne Métropole et de la région Rhône-Alpes. Le fonds photographique est consultable sur la plateforme VIVA ARTS à l'adresse suivante : https://viva-arts.univ-st-etienne.fr. Les illustrations de cet article sont la propriété du Musée d'art moderne et contemporain de Saint-Étienne Métropole, reproduites avec l'aimable autorisation de Françoise Cestac-Haeffelin.

Qu'elles soient documentaires, publicitaires, journalistiques ou artistiques, les représentations du travail sont révélatrices de préoccupations sociétales; elles témoignent de l'évolution des métiers et des changements liés aux transformations techniques et économiques. L'étude du monde du travail gagne à considérer les divers objets de la société visuelle. L'appréhension des différentes pratiques commerciales de représentation permet notamment de repenser l'industrialisation et le capitalisme (Deluermoz et al., 2014). Le rapport à la marchandise se tisse en effet, en partie, dans les processus de circulation de la publicité dont les formes de mise en images et de spectacularisation sont multiples et variées : affiches, prospectus, dépliants, encarts, 
spots vidéo et radiophoniques, mais aussi salons et foires, devantures et étalages de commerces...

L'histoire de l'art s'est longtemps limitée à la prise en compte d'œuvres reconnues, sanctionnées par certains modes d'exposition ou de publication. Jusqu'à une période récente, peu de considération était accordée aux arts appliqués et travaux de commande. Depuis le début des années 1990, le courant de recherche des Visual Studies qui émerge dans les pays Anglo-saxons contribue à pallier ce manque: il englobe les arts et la culture visuelle au sens large et aborde les processus de production et de circulation des représentations.

« ̀̀ un moment où le flux des images et leur puissance suscitaient de nouveaux questionnements, ce « champ mouvant » proposait de saisir non seulement l'image dans toutes ses dimensions et supports, mais la "fabrique visuelle de[s] société[s] », les dispositifs de vision et les modalités du regard. Une telle ambition supposait de faire dialoguer l'histoire, l'histoire de l'art, la sociologie, l'anthropologie, la philosophie, la psychanalyse, etc. » (Ibid., 139)

Dans cette optique, l'« examen attentif des médias et des autres éléments constitutifs de la culture de masse nous aide à mieux comprendre la société » (Hoggart, 2013, 91-92) : l'art de masse est en effet «davantage susceptible d'avoir recours à des schémas, des signes ou des codes communs que les œuvres d'imagination individuelle " (Ibid., 95) ; il fournit un riche enseignement sur le public, les modalités de réception et sur la manière dont les représentations influent sur la société.

La photographie - médium d'expression privilégié d'une esthétique populaire, qualifié d'«art moyen» (Bourdieu, 1965) - constitue un objet d'étude intéressant. La production et l'utilisation d'images photographiques se subordonnent à des fonctions sociales (Freund, 1974) ${ }^{1}$. La recherche gagne à prendre en compte les images de commande - pour la publicité (Gastaud, 2006), la presse, l'édition, l'industrie, la mode... - et, d'une manière plus générale, à considérer le marché et les usages de la photographie (Denoyelle, 1997). Le fonctionnement des agences et studios photographiques, ainsi que les modalités de production de la publicité moderne, ont été peu étudiés. En sortant des pratiques reconnues pour aller vers ce type de représentations de masse, soulevant de nombreux enjeux sociétaux, la connaissance des images du travail ne peut qu'être améliorée.

\section{Le fonds des Éditions Paul-Martial}

5 Au début du XXe siècle, alors que la publicité en est à ses balbutiements (Chessel, 1998), le contexte propice de l'industrialisation entraîne une nouvelle consommation à laquelle il faut apporter une visibilité (Méaux et Dancer-Mourès, 2014). Les grandes sociétés commencent à miser sur la communication. En 1926, Paul-Martial Haeffelin crée les Éditions Paul-Martial, société d'édition publicitaire qui deviendra l'une des plus reconnues dans le domaine. L'entreprise se spécialise dans l'industrie ${ }^{2}$ et propose à ses clients plusieurs types de prestations comme la conception de lignes graphiques, logos et affiches, d'encarts publiés dans les journaux et magazines, l'édition d'imprimés publicitaires et la conception de vitrines et stands d'exposition. Ses locaux parisiens sont dotés d'ateliers de dessin et typographie, d'une imprimerie et d'un studio photographique ${ }^{3}$. 
6 Les photographes de l'entreprise se déplacent également sur certains sites industriels pour la réalisation de reportages ${ }^{4}$ : l'ensemble de l'activité de l'entreprise commanditaire est alors photographié, depuis l'extérieur des usines jusqu'aux objets produits, en passant par les machines, les différentes étapes de la production et les ouvriers au travail. Ces images sont ensuite insérées dans des catalogues, brochures et dépliants publicitaires. Les Éditions Paul-Martial réalisent près de quarante-sept mille photographies - de reportage et de studio - entre 1929 et $1968^{5}$.

7 Une partie des archives de l'entreprise a été vendue aux enchères en $1991^{6}$ mais la grande majorité a disparu. Les documents sauvegardés sont aujourd'hui conservés dans trois collections institutionnelles et une privée: celle du musée d'art moderne et contemporain de Saint-Étienne Métropole (MAMC+) qui comprend deux mille huit-cent quarante-deux tirages sur papier baryté au gélatino-argentique, onze planches de tirages contact, cent-quarante-huit négatifs sur films celluloïd souples, cinq sur plaques de verre, une maquette originale, vingt-cinq publications éditées par l'entreprise ainsi que plusieurs documents administratifs tels que des répertoires ou carnets référençant la production de la société ; celle du département des estampes et de la photographie de la Bibliothèque nationale de France qui compte près de douze mille deux cents négatifs sur films souples, mille cinq cent sur plaques de verre et soixante-douze tirages; celle du Kunstmuseum de Bâle comprenant quatre cent soixante-quinze tirages et celle de l'expert et marchand d'art Marc Pagneux qui compte près de trois mille tirages, quelques imprimés et carnets de référencement des négatifs.

Malgré le nombre important de documents conservés, l'étude est fortement contrainte par la disparition d'une partie des archives de l'entreprise. Les milliers d'images qui composent les différents fonds connus constituent, en effet, moins du tiers de la production photographique totale de l'entreprise. Les réalisations publicitaires de l'entreprise étant conçues pour être diffusées, il est nécessaire de confronter les photographies aux divers supports de médiatisation afin d'en préciser les enjeux. Malheureusement, rares sont également les brochures et catalogues publicitaires réalisés par l'entreprise - dans lesquels les photographies sont publiées - qui demeurent aujourd'hui. Ces publications constituent donc des objets précieux, qui permettent de comprendre les modalités d'insertion et les conditions de réception des images.

\section{Quelles images pour quelles visées?}

9 Les archives des Éditions Paul-Martial constituent une source privilégiée pour appréhender les modalités du travail au début $\mathrm{du} \mathrm{XX}^{\mathrm{e}}$ siècle et les représentations publicitaires qui les accompagnent. L'enquête sur les usages de ce type d'images est indispensable : il faut appréhender la nature des commandes, les conditions de prise de vue et les modalités de diffusion des photographies pour en comprendre les finalités. Les documents administratifs renseignent les différents prestataires auxquels l'entreprise a pu faire appel, mais aussi la nature des commandes - affiche, dépliant, brochure, encart, prospectus... Ils permettent en outre d'identifier l'entreprise commanditaire, le photographe et de dater les images. L'étude des négatifs permet quant à elle de mieux cerner le fonctionnement de l'entreprise et les conditions de production des photographies: leur nature, les processus de réalisation et le type de matériel utilisé. 
10 Les photographes des Éditions Paul-Martial doivent proposer des images conformes à la requête du client. La visée publicitaire conditionne la réalisation de photographies constituant une forme de marchandise industrielle : les images répondent à un cahier des charges et permettent un échange monétaire ; le traitement que le photographe fait subir au photographié est toujours médiatisé par le type de relation qui l'unit à son client (Bourdieu,1965, 20). Il s'agit donc de considérer les enjeux relatifs au témoignage d'un reportage relevant d'une commande publicitaire: celui-ci relève de demandes précises de la part des entreprises qui sollicitent l'obtention d'images gratifiantes de leur activité ; l'autocritique est rejetée au profit du regard valorisant. C'est une vision nécessairement positive de l'activité et des conditions de travail qui est retranscrite. Le photographe Ito Josué explique à ce sujet :

«Historiquement, à partir du moment où la photo documentaire est devenue publicitaire [...] toutes les photos se ressemblent : il n'y a pas d'ouvriers qui soient à des tâches pénibles et d'autres à des tâches privilégiées; ils sont tous " heureux ", dans des usines "très sophistiquées ", même si elles ne le sont pas : on repeint les murs de manière très propre, les machines sont impeccables, les costumes sont toujours tout neufs. Bien sûr, il n'y a aucune blessure apparente sur l'ouvrier que l'on va représenter, ce serait la fin des fins; ils sont tous heureux, ce qui implique, socialement, qu'ils sont tous bien payés. Voilà un problème très grave... [...] On connaît le principe de la pub: ce qu'on nous demande c'est de les représenter heureux, avec un travail bien payé, agréable, pas de stakhanovisme, «Taylor? Connais pas ». » (Josué, 1996, 24)

11 Les reportages photographiques des Éditions Paul-Martial ne rendent donc pas seulement compte des pratiques du travail de l'époque; ils illustrent également la volonté promotionnelle des industriels. 
Image 1

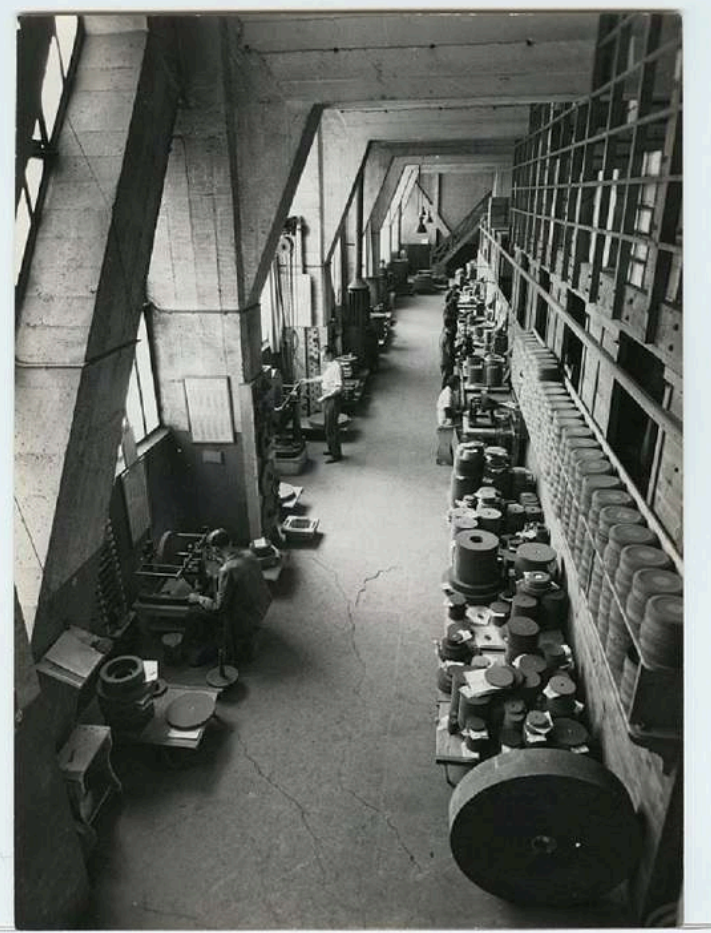

Éditions Paul-Martial, reportage pour la Compagnie Centrale des Émeris et Tous Abrasifs (CETA), vue partielle de l'atelier de réception et de contrôle des meules terminées, 1939, papier baryté au gélatinoargentique, $24 \times 17 \mathrm{~cm}, \mathrm{n}^{\circ}$ inv. 2007.30.0130.

Collection Musée d'art moderne et contemporain de Saint-Étienne Métropole, avec l'aimable autorisation de Françoise Cestac-Haeffelin 
Image 2

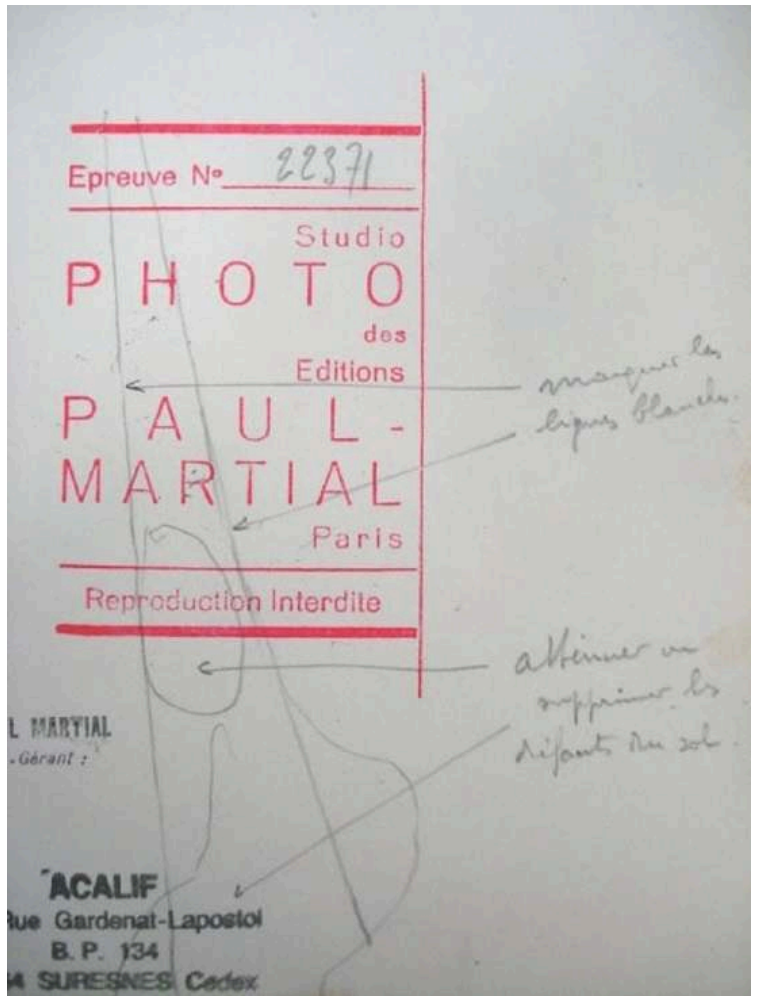

Revers du tirage.

12 Si les usines peuvent constituer le lieu d'une théâtralisation, les photographes n'hésitent pas également à manipuler les images une fois en laboratoire. Les annotations portées au dos de certains tirages indiquent d'éventuelles retouches : des directives sont parfois données pour des retirages afin d'améliorer la qualité du rendu, de gommer certains éléments ou de recadrer les images en vue de la publication. Cette vue intérieure d'usine réalisée dans le cadre d'un reportage pour la Compagnie Centrale des Émeris et Tous Abrasifs (CETA) en atteste. Des directives en termes de retouche figurent au dos du tirage: un tracé au crayon reprend la composition de la photographie; certaines zones pour lesquelles des modifications sont demandées y sont désignées avec les indications "marquer les lignes blanches » et "atténuer ou supprimer les défauts du sol». Ces annotations manifestent la volonté d'une modification de certains aspects de l'image pour la publication dans le but de magnifier le sujet. 


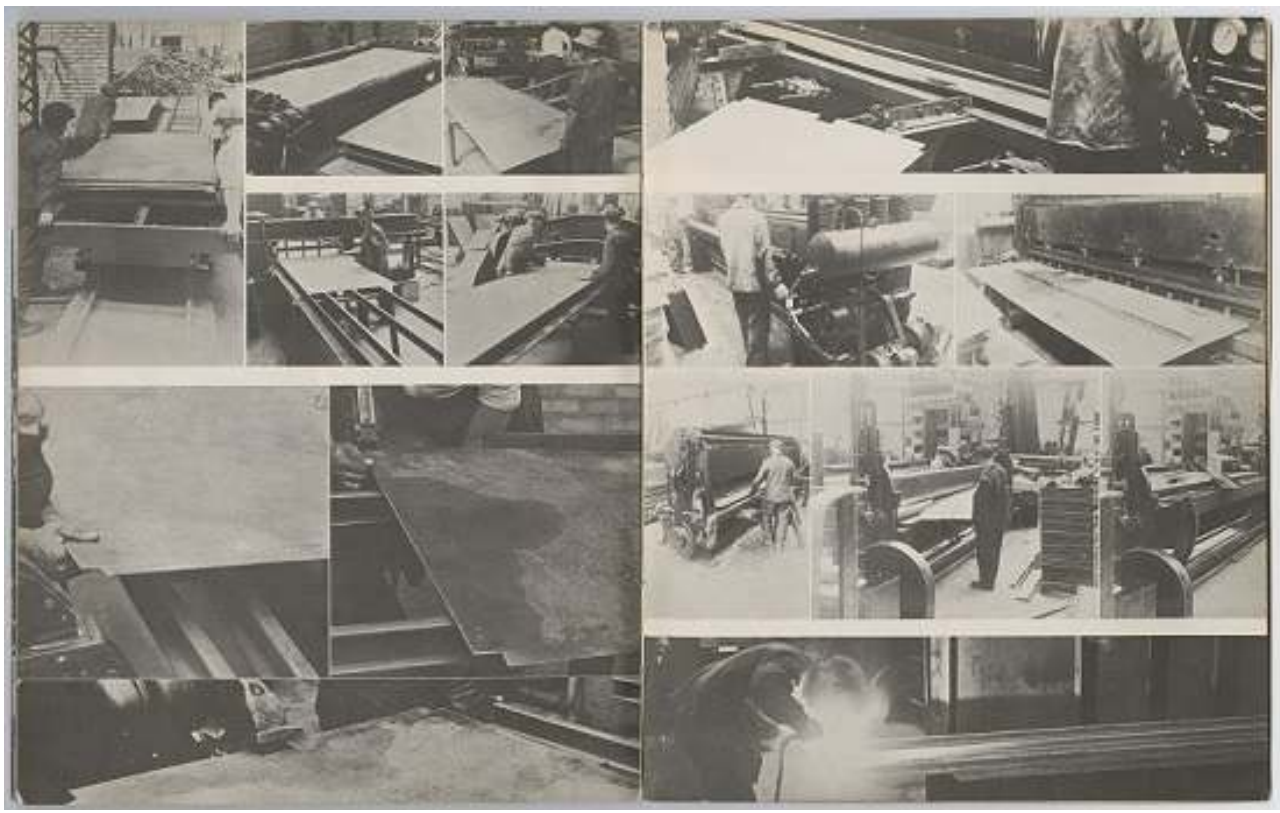

Double page intérieure du catalogue Les maisons des Constructions métalliques Fillod, Paris, Éditions Paul-Martial, vers 1931.

Collection Musée d'art moderne et contemporain de Saint-Étienne Métropole, avec l'aimable autorisation de Françoise Cestac-Haeffelin

13 Cet exemple de double page intérieure du catalogue des Constructions Métalliques Fillod (CMF) est symptomatique des diverses manipulations de recadrage et d'agencement des images réalisées pour la page imprimée. Cette publication découle d'un reportage réalisé en août 1931 sur les usines de fabrication de l'entreprise à Florange (Moselle) par le photographe des Éditions Paul-Martial, Gaudillère. Le regard de ce dernier se porte sur l'aménagement intérieur des ateliers, sur les machines et outils employés, mais aussi sur les différentes étapes et méthodes du travail illustrées par les manipulations et gestes des ouvriers. L'absence de légendes qui aideraient à identifier les sujets représentés se couple à une recherche de composition de la page imprimée : la photographie a ici davantage une vocation illustrative et esthétique que documentaire.

14 La composition de ces images est dynamisée par de nombreuses lignes de force obliques qui visent, pour beaucoup, à amplifier la longueur des machines et des plaques de tôle. En optant pour des angles de prise de vue de trois-quarts, qui donnent à voir la perspective des lieux et des engins, le photographe confère une impression de grandeur aux sujets. Certaines images constituent des plans rapprochés sur les gestes et manipulations des ouvriers, focalisant de cette manière l'attention du regardeur sur l'action en cours. Sur l'image en bas de la page de droite, la lumière provoquée par la soudure crée un fort contraste avec l'obscurité environnante de l'atelier, renforçant l'importance et la dangerosité du travail. L'application de l'ouvrier dans cette tâche rigoureuse est ainsi glorifiée. Les photographies ont été recadrées dans un second temps afin de polariser le regard du spectateur sur certains éléments. Cette pratique photographique atteste d'une réelle étude de la composition, que ce soit lors de la prise de vue ou au moment d'agencer la page imprimée. 


\section{Enjeux de l'étude des sources : de la vision du travail à celle de l'expérience figurative} commerciale. Bien que relevant de commandes, ces images et publications sont pourtant propices à une recherche esthétique. L'« expérience esthétique " ne relève en effet pas exclusivement du domaine de l'art et peut s'appliquer à différents types d'objets (Schaeffer, 1996, 128). Dans le même temps, un même objet - relevant par exemple des arts appliqués - peut correspondre, de la part de son auteur, à des intentions de diverses natures: fonctionnelles et mercantiles, mais également esthétiques, ces visées n'étant pas exclusives les unes des autres. Pour saisir les différents processus par lesquels les images sont produites et consommées, il s'agit d'appréhender le faisceau de relations qui s'y rapporte (Becker, 1982), à travers une mise en conversation de la technique, l'économie, l'histoire, la sociologie, l'histoire de l'art et l'esthétique. d'importantes avancées industrielles et techniques. Celles-ci modifient et redéfinissent les modalités du travail, mais influent également sur la création plastique. L'art use de nouvelles techniques et aborde de nouvelles thématiques : les cubistes s'inspirent des formes géométriques des produits manufacturés pour développer un langage plastique basé sur la schématisation ; les puristes représentent les objets de la vie quotidienne en valorisant les formes simples et la machinerie; les futuristes italiens et les constructivistes russes exaltent le progrès et les avancées techniques; les précisionnistes américains s'attachent aux formes mécaniques ainsi qu'aux paysages urbains et industriels qu'ils représentent par des compositions géométriques simplifiées aux lignes de force marquées; Marcel Duchamp exploite la productivité naissante et invente la notion de ready-made.

L'homme est le plus souvent exclu de ce type de représentations. Lorsqu'il est figuré, il est soumis à une même schématisation que l'univers des objets inanimés. Il en va ainsi du bûcheron peint par Kasimir Malevitch en 1912, réduit à un assemblage de cylindres et de cônes rappelant les tuyaux et entonnoirs géométriques de la mécanique industrielle, jusqu'à se fondre avec l'arrière-plan abstrait de la toile. Le corps du mécanicien de Fernand Léger (1920) fait preuve d'une même simplification. L'artiste adaptera ce traitement qualifié de "tubiste » à ses constructeurs au début des années 1950. Dans la lignée de la conception de l'homme-machine, le corps humain est alors perçu, au même titre que l'ensemble des éléments du monde, comme un agencement de formes géométriques.

La photographie d'art accompagne cette tendance : dans les années 1920 en Allemagne, les photographes de la Nouvelle Objectivité s'intéressent notamment aux objets quotidiens ; en France, les acteurs de la Nouvelle Vision font preuve d'un engouement pour les thèmes emblématiques de la modernité. Ils usent pour cela des différentes capacités techniques de la photographie: les appareils de petit format, alors récemment arrivés sur le marché, favorisent l'utilisation d'angles de prise de vue basculés et de plans rapprochés. Les techniques du photomontage, de la solarisation et de la surimpression sont aussi régulièrement utilisées pour expérimenter. Toutes ces mutations des formes de l'expérience figurative sont contemporaines des évolutions techniques.

Images du travail, travail des images, 8 | 2020 
19 Si le regard de ces photographes se porte le plus souvent sur les machines, architectures et objets du quotidien, certains placent l'homme au cœur des représentations. Il en va ainsi de François Kollar qui réalise une campagne photographique d'envergure sur le monde du travail et de l'industrie entre 1931 et 1934 pour le compte des éditions Horizons de France. Près de dix mille vues sont prises sur deux cent sites du territoire français. 1900 d'entre elles sont publiées au sein des quinze volumes de La France travaille. Les albums font la part belle aux machines et structures industrielles. Mais c'est avant tout l'homme au travail et le langage des gestes qui retiennent l'attention du photographe (Bachollet et Lelieur, 1986; Rivallin et Viewing, 2016). De son côté en Allemagne, August Sander réalise à la fin des années 1920 des centaines de portraits d'hommes et de femmes dans un style documentaire (Lugon, 2001) qu'il rassemble sous l'intitulé «Hommes du XXe siècle». L'ensemble, qui constitue une mosaïque des visages de l'époque, est divisé en plusieurs catégories parmi lesquelles trois sont consacrées aux travailleurs: "paysans ", "artisans » et « catégories socioprofessionnelles».

En France, un certain nombre de ces photographes d'avant-garde travaillent pour la presse, l'édition, la publicité, la mode et l'industrie en parallèle de leurs recherches personnelles (Bouqueret, 1997), mêlant les pratiques sans les hiérarchiser (Fleig, 1997, 182). Bien souvent, la commande constitue pour eux une occasion d'expérimenter et de mettre en pratiques leurs recherches formelles. Certains photographes aujourd'hui reconnus, tels qu'André Papillon, André Kertész, Henry Lachéroy, Roger Schall et François Kollar, furent d'ailleurs associés aux Éditions Paul-Martial'7 . La production de l'entreprise rejoint certains codes esthétiques de la modernité artistique : l'ambition de clarté et de lisibilité pour certains objets isolés, la présentation de machines par des cadrages serrés, l'utilisation de la contre-plongée pour la représentation des architectures ou encore le goût pour la série... La technique du photomontage est également exploitée pour des compositions qui sont intégrées dans des affiches, des catalogues ou présentées sur les murs de stands d'exposition. Ces parentés sont révélatrices d'importants échanges entre les pratiques publicitaires et le champ de l'art durant l'entre-deux-guerres.

21 Ce type de travaux de commande, qui se sont développés en même temps que des pratiques artistiques plus reconnues, ont eux aussi contribué à forger la culture visuelle de leur temps. L'examen du fonds d'archives des Éditions Paul-Martial apporte un regard nouveau sur les pratiques photographiques et artistiques de cette période. Si ces représentations permettent de constater les nombreuses évolutions qui ont lieu dans le domaine du travail et de la production au début du $\mathrm{XX}^{\mathrm{e}}$ siècle, elles permettent également de percevoir les mutations qui ont eu lieu au même moment dans les formes de l'expérience figurative.

Le regard porté sur ces objets amène à penser une importante porosité entre les champs, à revisiter les catégories et à reconsidérer certaines hiérarchies établies dans l'histoire de la photographie. Il s'agit de réévaluer l'importance des entités collectives qui ont œuvré à la commercialisation des produits de l'industrie - telles que les studios et agences de publicités - et de revoir certaines idées reçues sur l'art et les avantgardes; il est important d'appréhender pour cela les liens qui s'établissent entre « art et technique " (Francastel, 1956) et "art et culture» et de repositionner les photographes et œuvres reconnus dans un contexte économique et social plus global. 


\section{Une mise en scène de l'homme au travail}

23 L'étude chronologique des photographies du monde du travail permet d'appréhender les évolutions des modalités de représentation. Les images réalisées à la fin du XIX ${ }^{e}$ siècle et au tout début du XXe siècle (Assegond, 2013) correspondent généralement à des scènes posées : les travailleurs arrêtent leur activité pour répondre à un « rendezvous photographique »; leurs postures sont rigides et leurs regards figés sur l'objectif. Après la Première Guerre mondiale, la présence des ouvriers est mise de côté au profit des intérieurs d'usines, des machines et des pièces isolées. C'est une productivité technique plus froide qui est illustrée : parfois l'homme pose à côté de la machine ou du produit usiné pour en indiquer la taille ; il est d'autrefois représenté dans son action du travail, mais de manière distanciée.

Un changement s'opère dans le courant des années trente avec l'apparition de photographies du travail en train de se faire, centrées sur la présence humaine. Il en va ainsi de certains reportages réalisés par les Éditions Paul-Martial pour J. J. Carnaud et Forges de Basse Indre (1930), la Société des aéroplanes Henry Potez (1932), Moët et Chandon (1936), la Société de houilles Delmas-Vieljeux et la Compagnie Centrale des Émeris et Tous Abrasifs (1939) qui rendent compte de l'activité de l'homme, de son rapport à la machine, ou encore des gestes du travail. Plusieurs éléments doivent être appréhendés: le point de vue du photographe pour illustrer l'action, les différentes postures des ouvriers qui peuvent être appliqués comme détachés du travail, leurs regards concentrés sur l'action qui s'accomplit ou qui convergent vers l'objectif, leur disposition en groupe ou éparpillée derrière chaque poste de travail, leurs gestes en mouvement ou arrêtés...

\section{Figuration}




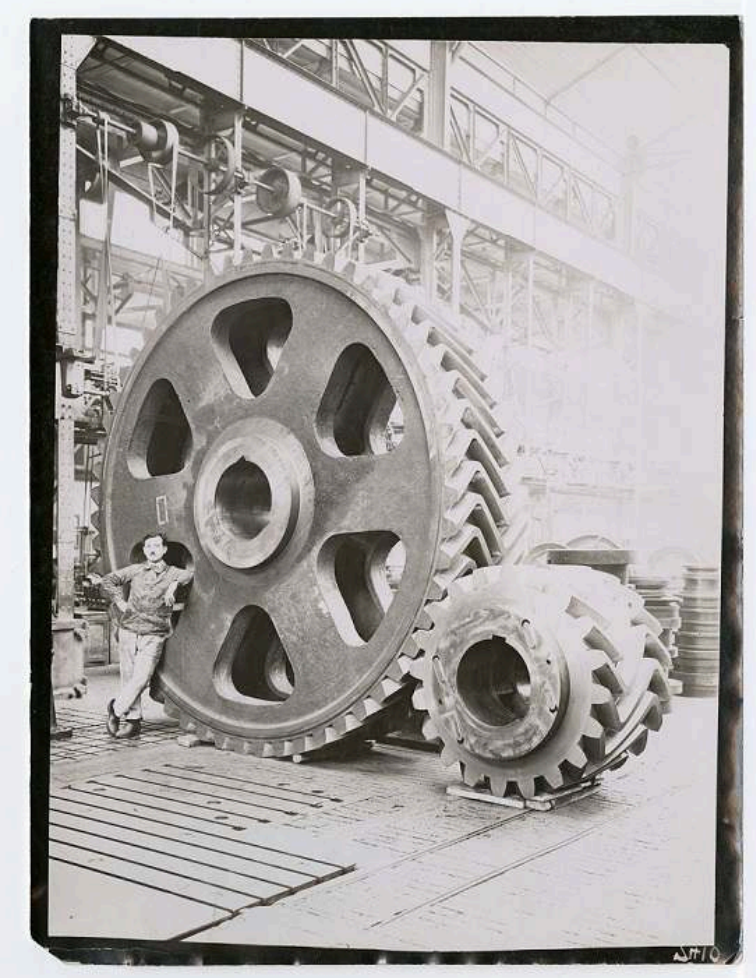

Éditions Paul-Martial, reportage de S. Nicolas pour la Compagnie des Forges et Aciéries de la Marine et d'Homécourt (FAMH) à Saint-Chamond (Loire), engrenage et ouvrier posant, novembre 1931, papier baryté au gélatino-argentique, $17,8 \times 23,8 \mathrm{~cm}, \mathrm{n}^{\circ}$ inv. 2007.30.0360.

Collection Musée d'art moderne et contemporain de Saint-Étienne Métropole, avec l'aimable autorisation de Françoise Cestac-Haeffelin

Régulièrement, les photographes confrontent les machines et pièces usinées à l'homme. Cette image prise pour la Compagnie des Forges et Aciéries de la Marine et d'Homécourt (FAMH) est symptomatique de ce type de scénographie : l'homme présent à côté de l'engrenage haut de quatre mètres ne se présente pas comme un ouvrier en action, mais comme un simple figurant se contentant de poser. Avec ce type de manipulation consistant à valoriser la fabrication usinière, la visée publicitaire est clairement assumée : l'ouvrier sert de "mètre-étalon " pour indiquer les proportions de l'outil ; il est instrumentalisé pour illustrer la puissance industrielle. Les anciens photographes d'usine en témoignent :

«M. Duboeuf. Je lui expliquais : «C'est pour donner une idée de la taille de la pièce, si vous voulez vous y mettre. " J'en mettais un petit de préférence.

Tous. Oui, on mettait le plus petit.

M. Duboeuf. Dans tous les ateliers on avait le préposé type ; on ne le mettait pas à chaque fois mais par moment c'est carrément le chef de service qui disait « Il faudra mettre...»

G. Faure. «Pas celui-ci parce qu'il n'est pas beau. »

M. Duboeuf. Dans un atelier, il y avait le grand Sagnal et le petit Morin et, suivant le cas, le chef de service me disait : « Tu prends ta photo avec le grand Sagnal » ou « tu la prends avec le petit Morin ». Le grand Sagnal, je ne me rappelle pas dans quelle circonstance j'ai pu le prendre, en fait je photographiais plus le petit Morin. Mais c'est vrai qu'on est obligé de varier aussi parce que sur les catalogues il ne fallait pas toujours mettre le même bonhomme ${ }^{8}$. » (Duboeuf, Faure et Frécon, 1996, 17-18) 
L'image publicitaire procède ainsi de la manipulation et de la déformation. La prise de vue suspend l'activité de l'usine qui se trouve neutralisée. Elle altère la réalité selon les vœux du commanditaire: l'atelier - qui est généralement lavé avant la venue de l'opérateur - devient un théâtre du monde industriel ; la mise en scène ne relève pas uniquement du photographe, mais d'un dispositif développé par un ensemble de participants. L'ouvrier bascule d'une position de travailleur actif à celle de figurant : il participe à une opération ne relevant plus de la production industrielle mais de l'acte photographique; il s'agit du « seul genre photographique où l'homme peut et doit faire face à l'objectif sans rien faire d'autre » (Peroni et Roux, 1996, 139).

\section{Image 5}

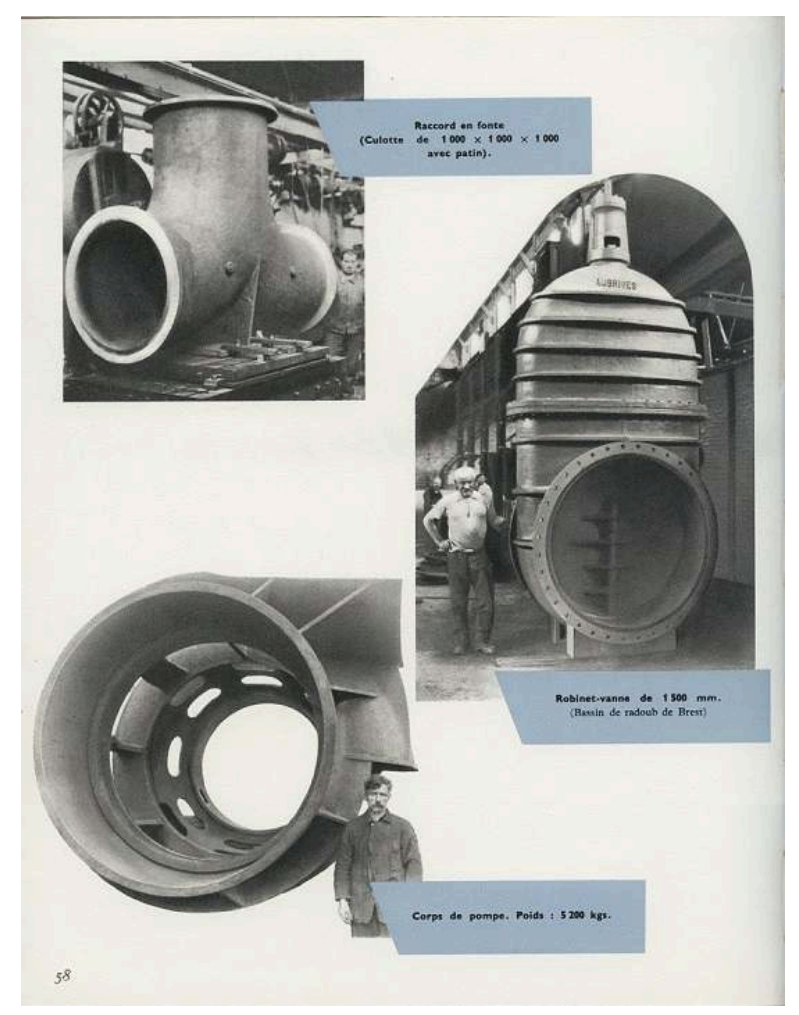

Page intérieure du catalogue publicitaire Schneider \& Cie, janvier 1955, Création et réalisation des Éditions Paul-Martial, Paris.

Le travail de mise en page réalisé pour la publication de ce type d'images renforce l'instrumentalisation. Sur la page du catalogue réalisé pour Schneider \& Cie, les légendes des photographies indiquent les caractéristiques des pièces industrielles uniquement, sans faire mention des hommes présents à leurs côtés : « robinet-vanne de $1500 \mathrm{~mm}$ »; « Corps de pompe. Poids : $5200 \mathrm{kgs}$ »; « Raccord en fonte (Culotte de $1000 \mathrm{x}$ 1000 x 1000 avec patin ». La présence ouvrière endosse ainsi un rôle informatif comparable à celui des légendes. Le texte complète les indications transmises par la mise en scène photographique.

\section{Épopée}




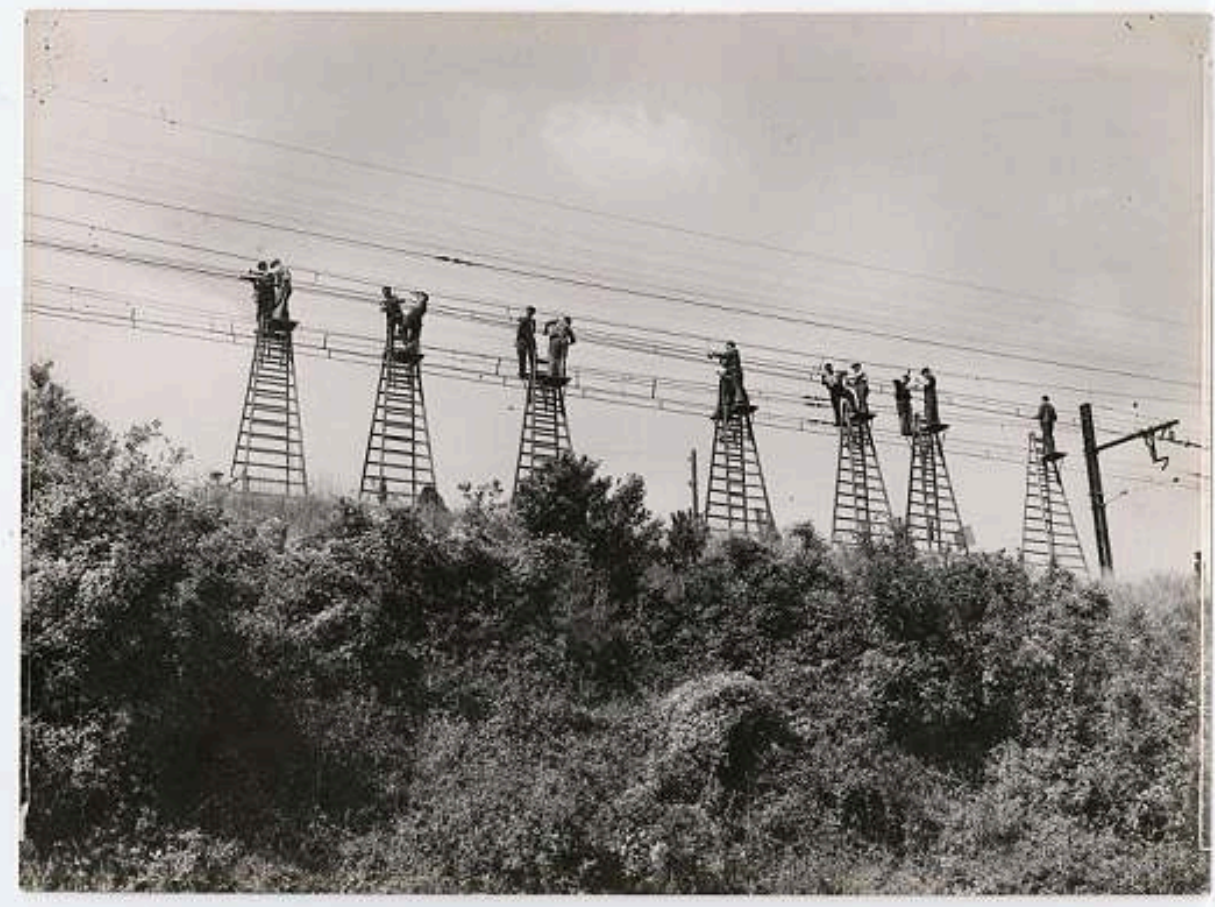

Éditions Paul-Martial, reportage pour la SNCF, ouvriers travaillant à la mise en place des caténaires, 1939, papier baryté au gélatino-argentique, 18 × 24,2 cm, nº inv. 2007.30.1100.

Collection Musée d'art moderne et contemporain de Saint-Étienne Métropole, avec l'aimable autorisation de Françoise Cestac-Haeffelin

Certaines représentations valorisent au contraire le savoir-faire humain, en atteste cette photographie qui met en avant la prouesse technique d'ouvriers de la SNCF installant des caténaires le long d'une voie ferrée. La contre-plongée accentue l'impression de hauteur des cheminots qui semblent accomplir une performance d'acrobates. Les échelles, dont l'équilibre semble précaire, nécessitent la contribution d'autres hommes au sol pour être stabilisées. À cela s'ajoute la dangerosité du contact avec les fils électriques. La disposition chorégraphiée des ouvriers le long de la ligne à haute tension rythme l'image. Le nombre important d'acteurs mobilisés dans cette opération témoigne d'une aptitude à travailler en équipe : la stabilité des cheminots perchés est garantie par les hommes à terre, chaque rôle ayant son importance dans l'opération en cours.

Tout comme la série d'images «Men at work» de Lewis Hine, qui chante les facultés incroyables du travailleur-héros, cette image est chargée de lyrisme: l'action des travailleurs semble relever de l'exploit. L'activité industrielle permet aux travailleurs de s'élever dans le ciel. Le travail humain est ainsi exalté : il semble incarner l'accès à la production et au progrès de toute une classe sociale. L'épopée ouvrière rejoint de cette manière celles de l'électrification et des grandes constructions, symboles d'innovation et des avancées de l'époque.

\section{Absorbement}




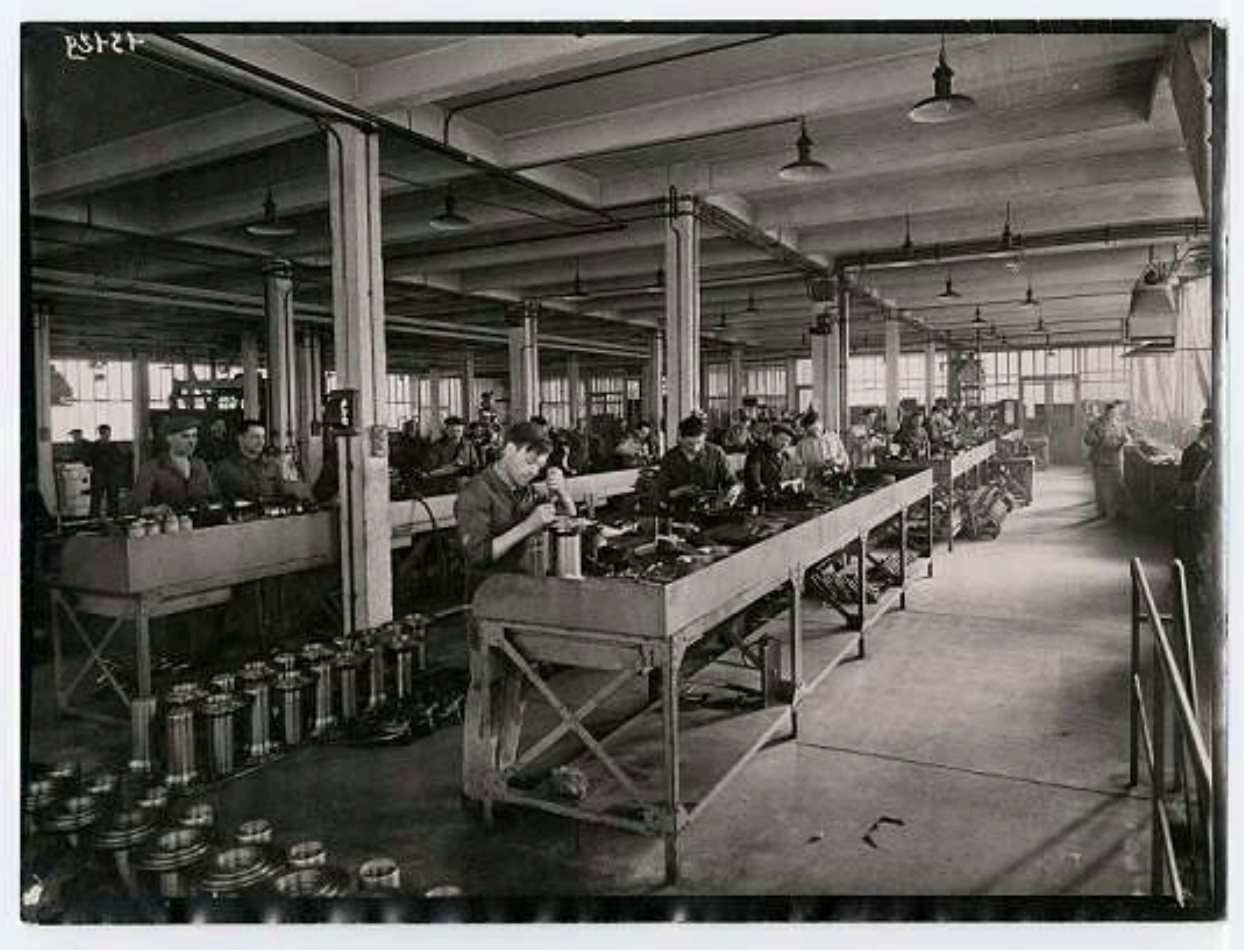

Éditions Paul-Martial, reportage de L. Bontemps pour la Précision moderne, ajustage, 1935, papier baryté au gélatino-argentique, $18 \times 24 \mathrm{~cm}, \mathrm{n}^{\circ}$ inv. 2007.30.36.

Collection Musée d'art moderne et contemporain de Saint-Étienne Métropole, avec l'aimable autorisation de Françoise Cestac-Haeffelin

Dans cette photographie d'usine de fabrication de matériel militaire, les travailleurs en action tiennent une place privilégiée. L'oblique qui s'étend du bas à gauche jusqu'en haut à droite met en avant la grandeur des lieux et le nombre important de postes de travail: aussi loin que s'étend l'atelier, l'activité occupe l'espace. Ce type de photographie donne l'impression d'une relative objectivité: l'image paraît correspondre à l'instant précis où le photographe a fait irruption dans la pièce; la scène aurait été transcrite sans que le cours des choses soit interrompu. Les ouvriers au premier plan semblent concentrés sur leur tâche au point d'en avoir oublié la présence de l'opérateur.

Michael Fried explique qu'« un personnage entièrement absorbé ou occupé par une action, un sentiment ou un état d'esprit est, par définition, indifférent à tout autre objet que celui de son absorbement » (Fried, 2013, 40). Il dresse un parallèle entre les photographies de Jeff Wall et les peintures de Jean Siméon Chardin dans lesquelles les personnages se trouvent immergés dans leurs activités et n'ont apparemment pas conscience de la présence d'un spectateur : ils ne « jouent » pas leur monde mais « sont simplement au monde ${ }^{9} »$. Les ouvriers de l'atelier de construction mécanique semblent de la même manière être " au travail ». Ce type de vue se veut ainsi non-théâtralisée : pris dans l'action de son activité, l'ouvrier ne perçoit pas le photographe - ou feint de ne pas le percevoir.

32 Pour Fried, cet état de concentration contribue à une position psychique positive : « l'absorbement est en soi parfaitement indifférent au statut général de ses objets ou de ses situations [...] ces actions spécifiques deviennent le vecteur d'un nouvel état mental 
ou spirituel, essentiellement "positif" " (Fried, 2013, 40). La photographie représente finalement autant l'action en train de se dérouler que l'intérêt des ouvriers pour leur travail. L'absorbement qui est donné à voir semble être synonyme d'une posture constructive: les travailleurs ne s'ennuient pas; leur activité contribue à leur épanouissement. L'effet naturel et spontané n'a donc d'autre but que de transcrire l'engagement des ouvriers envers leur activité.

Image 8

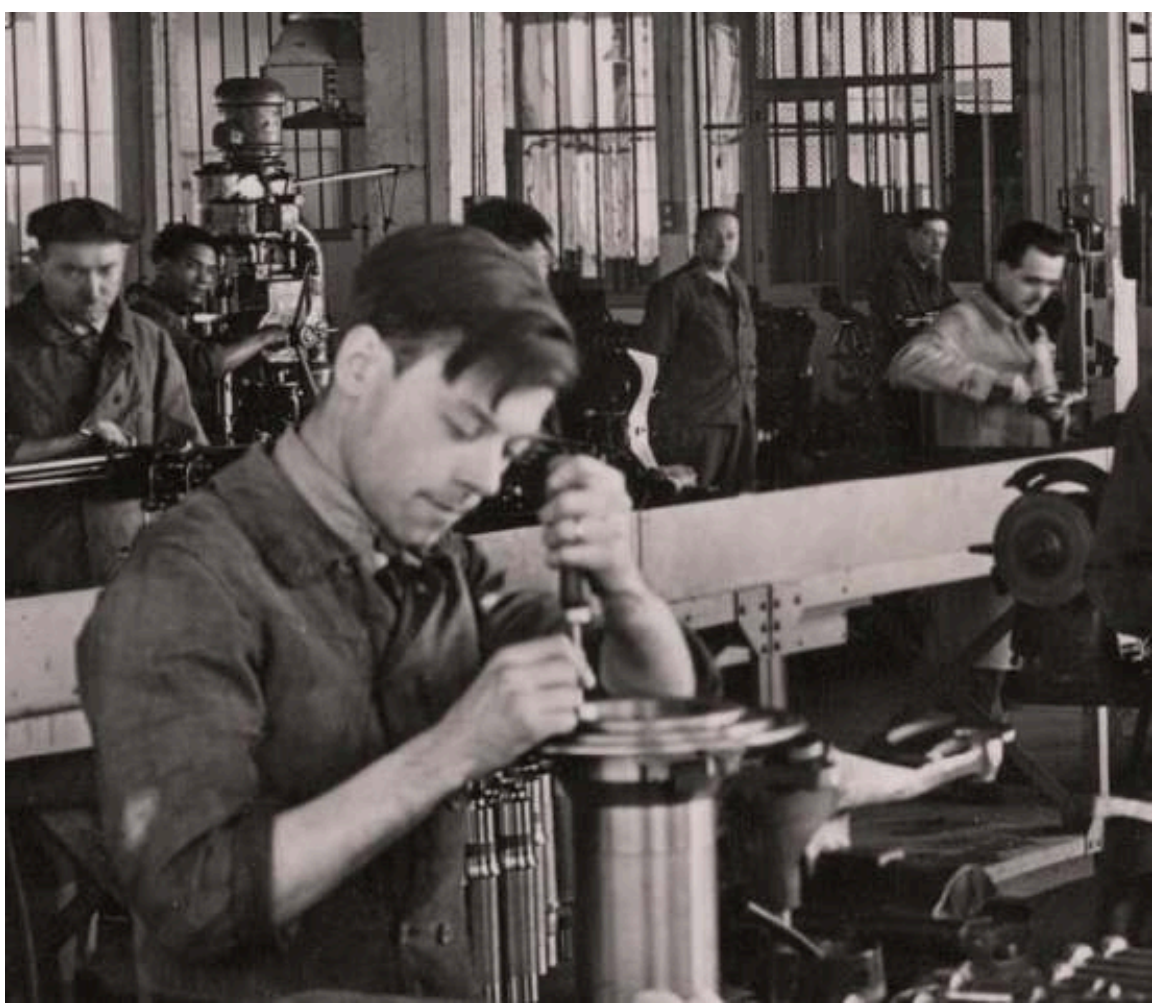

Détail de l'image

En étudiant l'image de plus près, il apparaît que les travailleurs présents à l'arrière-plan détournent le regard de leur tâche pour observer le photographe. Bien que se situant dans le viseur, ils semblent considérer que la distance qui les sépare de l'opérateur leur permet de ne pas être vus. Parmi ces "regards caméra ", se trouvent ceux des contremaîtres, eux aussi à leurs postes. Au lieu d'observer les ouvriers - ce qui serait vu comme une attitude trop explicitement répressive -, ils fixent, faute de mieux, le photographe, comme si celui-ci allait être un nouveau facteur de perturbation de l'ordre établi. En donnant ainsi l'air de regarder quelque chose, ils se donnent une contenance et rejouent leur rôle de chef d'atelier: ils semblent opérer, comme à l'habituel, par le regard; ils donnent l'impression de diriger la bonne manœuvre et le cours des choses par leur vision.

34 La photographie constitue donc à la fois un facteur de division entre les différents acteurs de l'atelier et l'objet d'une entreprise commune de figuration et de donation de sens au travail :

«[...] le photographe qui, par l'opération de cadrage, délimite une zone d'activité ; les représentants de l'encadrement, ingénieurs, contremaîtres, qui donnent les consignes et font respecter l'ordre du travail en train d'être photographié; les 
travailleurs présents dans le champ, qui tout en respectant le système des consignes, jouent leur propre rôle. » (Peroni et Roux, 1996, 101)

\section{Théâtralité}

Fried oppose les images de ce «mode absorbé » à celles d'un "mode théâtral ». Il qualifie les représentations de "presque documentaires » lorsque la scène est posée et que les acteurs adoptent une position et des gestes arrêtés : la fiction est renforcée pour constituer une illusion de réalité. Il est parfois difficile de différencier la reconstitution $\mathrm{du}$ pris sur le vif. Il s'agit de percevoir les éventuels indices d'un agencement scénographique et de déterminer si la photographie est ostensiblement théâtrale.

\section{Image 9}

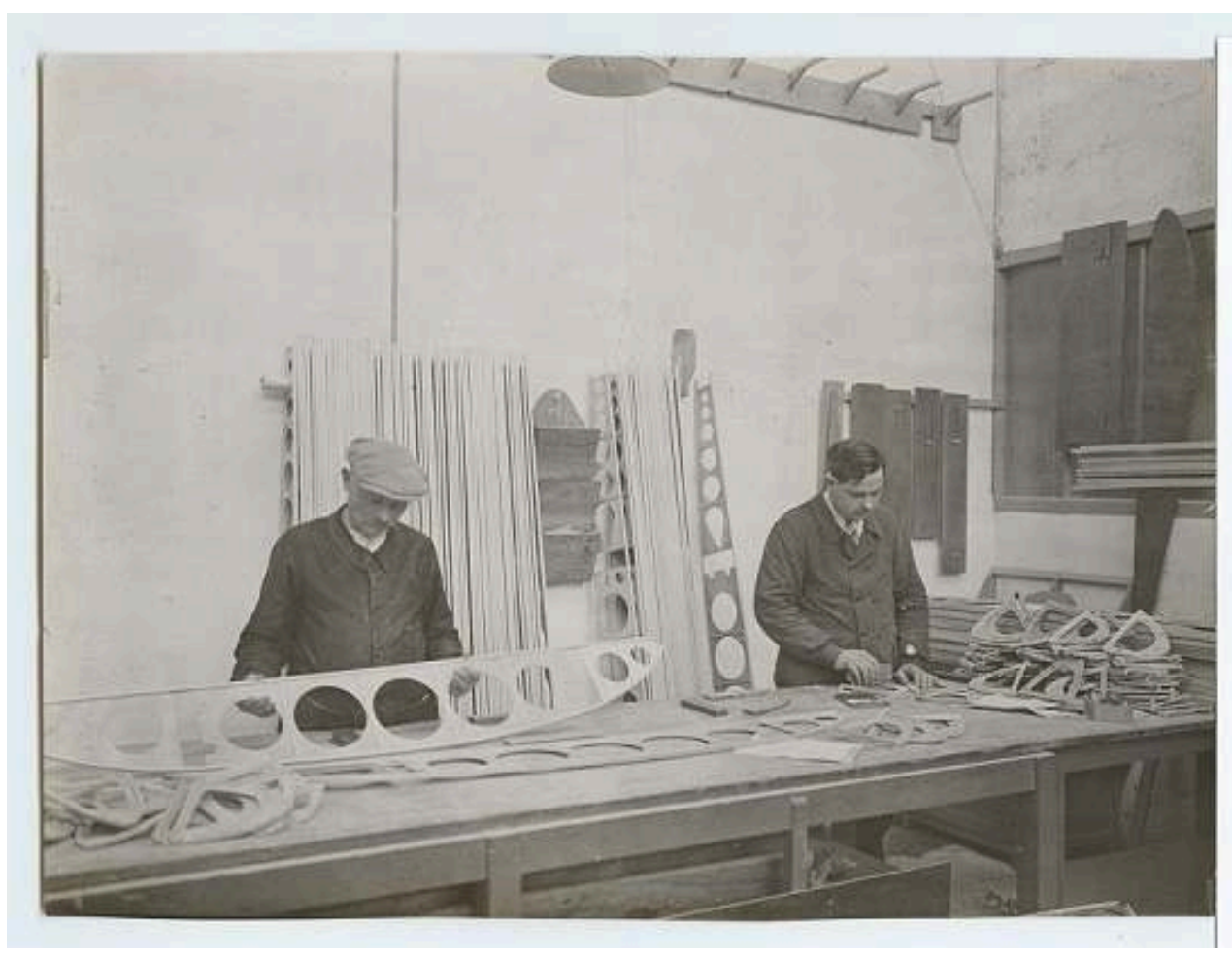

Éditions Paul-Martial, reportage pour la Société des aéroplanes Henry Potez, ouvriers au montage des ailes d'avions, 1932, papier baryté au gélatino-argentique, $17 \times 22,5 \mathrm{~cm}, \mathrm{n}^{\circ}$ inv. 2007.30.128.

Collection Musée d'art moderne et contemporain de Saint-Étienne Métropole, avec l'aimable autorisation de Françoise Cestac-Haeffelin

Cette image réalisée pour la Société des aéroplanes Henry Potez relève de cette seconde catégorie: l'image paraît moins spontanée que la vue précédente; le temps semble arrêté et les ouvriers en train de poser; ils ont vraisemblablement suspendu leurs gestes un instant à la demande de l'opérateur. Le regard baissé, l'ouvrier de gauche tient et observe un élément de montage sans le manipuler. Celui de droite a posé l'une de ses mains sur l'établi, tandis que la seconde maintient une pièce en bois. Les travailleurs simulent clairement l'absorbement : leur posture dramatisée est assumée ; ils jouent leur rôle. 


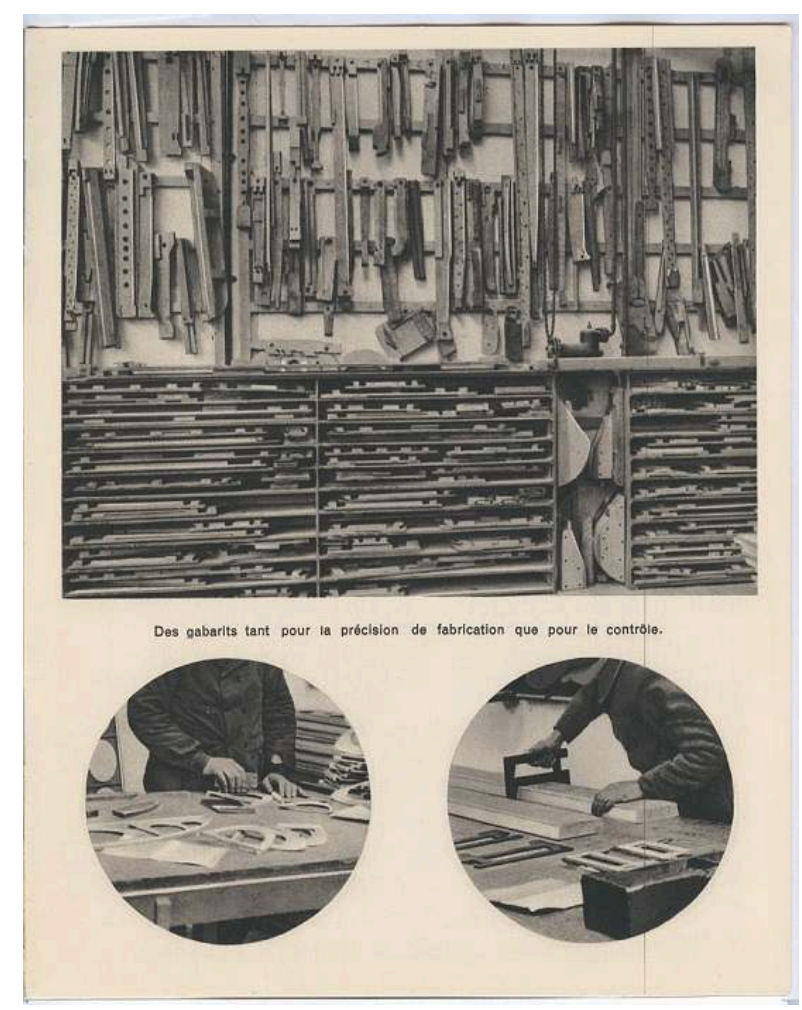

Double page intérieure du catalogue Avions Henry Potez, Collection des Bulletins Techniques de la Société des aéroplanes Henry Potez, n 16 bis, décembre 1932, création publicitaire et graphique des Éditions Paul-Martial.

Collection Musée d'art moderne et contemporain de Saint-Étienne Métropole, avec l'aimable autorisation de Françoise Cestac-Haeffelin

Les modalités de publication des images peuvent apporter des indices quant à une éventuelle mise en scène. Dans le cas de cette photographie, seul un détail de l'image, recadré en un format arrondi, est conservé. Une légende indique : "Des gabarits tant pour la précision de fabrication que pour le contrôle ». Il s'agit donc d'illustrer l'objet et son action plutôt que l'activité des ouvriers qui en usent. Pour cela, la démonstration se fait conjointement par le texte et l'image. Le recadrage focalise l'attention du lecteur sur l'outil et son utilisation; en coupant les corps des travailleurs, il crée une dépersonnalisation: les mains qui accompagnent les objets deviennent de simples outils techniques; les gabarits auraient tout à fait pu être représentés isolément, comme dans le cas de la photographie reproduite en haut de cette page du catalogue.

\section{Pédagogie}

Outre la volonté promotionnelle, l'image permet également parfois de conserver une trace de l'activité ou de préserver un savoir-faire spécifique. Il en va ainsi de certains gros plans de mains d'ouvriers qui visent à illustrer avec clarté les étapes d'un processus de travail. Ce type de photographie, qui requiert la pose de l'ouvrier, parvient à retracer une situation avec précision. 


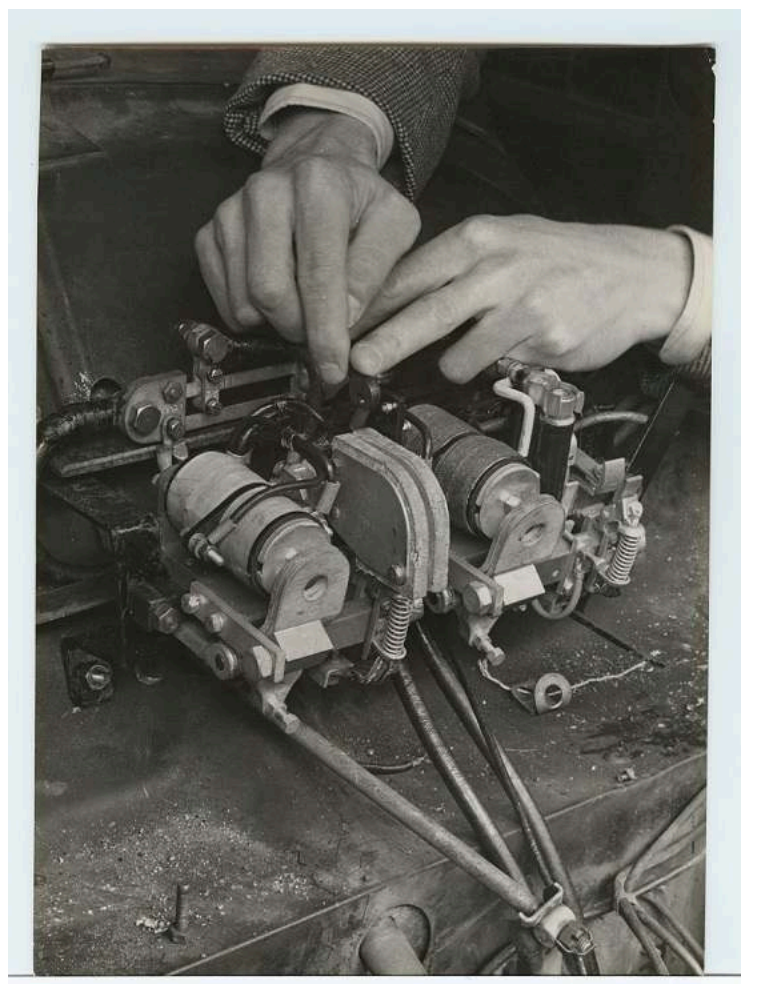

Éditions Paul-Martial, mains manipulant un mécanisme, vers 1939, papier baryté au gélatinoargentique, $23,5 \times 17 \mathrm{~cm}, \mathrm{n}^{\circ}$ inv. 2007.30.1016.

Collection Musée d'art moderne et contemporain de Saint-Étienne Métropole, avec l'aimable autorisation de Françoise Cestac-Haeffelin

Cette image de 1939 se veut didactique ; elle montre la manipulation à effectuer pour le réglage d'une machine. Elle relève d'une mise en scène assumée: le maniement du mécanisme ne doit pas être dissimulé par la main ou l'ombre du sujet ; l'ouvrier a placé ses doigts de façon à ce que l'opération soit clairement visible et compréhensible. Ce type de photographie est vraisemblablement destiné à une utilisation en interne des sociétés plutôt que pour une promotion publicitaire grand public. Le didactisme de ces vues, à même d'expliciter l'utilisation des machines, laisse imaginer une intégration au sein de manuels techniques.

La photographie semble transmettre une information que l'écrit ne saurait apporter :

«En s'approchant davantage, à la suite du photographe, les gestes apparaissent, simples ou complexes mais toujours efficaces. Aucun manuel technique ne saurait rendre la position du corps, le mouvement des mains. D'ailleurs, cela ne s'enseigne pas mais s'apprend sur le tas. » (Woronoff, 2003, 10)

41 Le gros plan réalisé pour ce type d'images coupe le corps de l'ouvrier qui est chosifié : devenu anonyme, il n'apparaît plus que comme un potentiel utilisateur de la machine, une annexe de l'outil. Ses mains, qui suffisent à illustrer sa présence, constituent une extension de l'outil. Elles «sont celles d'une personne quelconque. L'homme comme entité corporelle n'a pas ici de pertinence : le découpage photographique configure une nouvelle entité : le segment humain fonctionnalisé par l'outil» (Peroni et Roux, 1996, 148). Il n'est donc plus question ici de l'homme au travail, mais d'un savoir acquis et d'un geste technique millimétré, que la photographie s'attache à retranscrire. 


\section{Conclusion}

$42 \mathrm{Au}$ début du XXe siècle, certaines sociétés misent sur des supports publicitaires innovants pour promouvoir leur production, s'inscrivant ainsi dans l'ère de leur temps marqué par l'industrialisation mais aussi par l'évolution des techniques de communication et le développement de la publicité au sein de laquelle la photographie acquiert une place grandissante. L'étude de la perception de l'industrie dans la publicité permet une approche du monde du travail, tout comme de l'histoire des formes et des imaginaires de la culture visuelle au sens large.

43 L'étude du fonds Paul-Martial rend compte des conditions de travail de l'époque à travers la disposition des lieux de travail, la nature des machines utilisées et les méthodes de production développées. Mais la commande dont relèvent ces travaux implique le conditionnement $\mathrm{du}$ regard $\mathrm{du}$ photographe et une approche inévitablement méliorative. Ainsi, ces images permettent avant tout de révéler la volonté promotionnelle et la conception du monde du travail et de l'industrie des entrepreneurs. Les différentes modalités de médiation utilisées sont révélatrices de la nature de la stratégie de communication: la mise en scène sert généralement à convaincre l'acheteur; la sublimation de l'activité à le séduire ; et la documentation didactique à l'informer et le rassurer.

Les Éditions Paul-Martial usent de différentes techniques scénographiques pour promouvoir l'industrie: la mise en scène au sein des usines qui vise à embellir la réalité ; les manipulations de retouche des vues ; et la composition de la page imprimée qui recourt à un agencement du texte et de l'image. Qu'elle soit réalisée directement sur le lieu de la prise de vue ou ultérieurement, la scénographie se met au service de la volonté promotionnelle. 


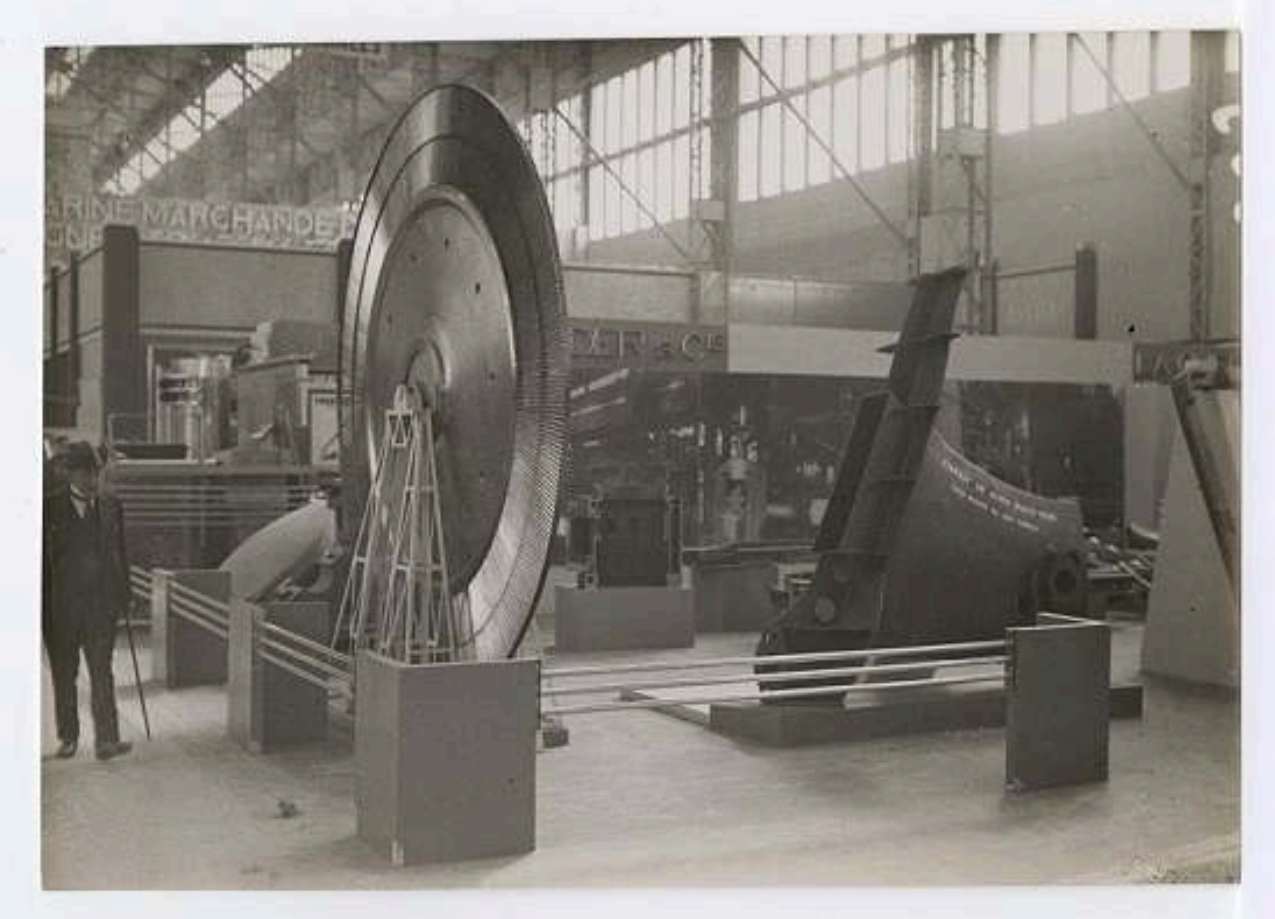

Éditions Paul-Martial, reportage sur la section de la grosse métallurgie de l'Exposition coloniale, stand Schneider \& Cie, février 1931, papier baryté au gélatino argentique, 23 x 16 cm, n inv. 2007.30.0090.

Collection Musée d'art moderne et contemporain de Saint-Étienne Métropole, avec l'aimable autorisation de Françoise Cestac-Haeffelin

L'entreprise s'illustre également dans une activité de conception de stands pour les foires et les salons industriels. Lorsqu'elle conçoit les différents espaces d'exposition de la section de la grosse métallurgie de l'Exposition coloniale en 1931 - pour Schneider \& Cie, l'Office Technique pour l'Utilisation de l'Acier (OTUA), les Aciéries de Longwy, la Compagnie des forges de Chatillon-Commentry et Neuves-Maisons, J. J. Carnaud et Forges de Basse Indre, la Société des Hauts-Fourneaux, les Forges et aciéries du Sautdu-Tarn - elle déploie la spectacularisation de l'industrie dans le champ tridimensionnel. La promotion de l'industrie s'effectue ainsi au travers d'une mise en scène permanente dont les diverses modalités d'expression font preuve d'une cohérence d'ensemble.

\section{BIBLIOGRAPHIE}

Assegond C. (2013), La Photographie du travail : usines, mines, chantiers (1850-1915). Analyse des modalités de représentation, thèse réalisée sous la direction de Michel Frizot, École du Louvre.

Bachollet R. et Lelieur A.-C. (1986), La France travaille. François Kollar : regard sur le monde du travail à la veille du Front populaire, Paris, Chêne. 
Becker H. S. (1982), Art Worlds, Berkeley, The University of California Press.

Bouqueret C. (1997), Des Années folles aux années noires : la nouvelle vision photographique en France, 1920-1940, Paris, Marval.

Bourdieu P., Boltanski L., Castel R. et Chamboredon J.-C. (1965), Un Art moyen. Essai sur les usages sociaux de la photographie, Paris, Minuit.

Chessel M.-E. (1998), La Publicité : naissance d'une profession, 1900-1940, Paris, CNRS, « Histoire contemporaine ».

Denoyelle F. (1997), La Lumière de Paris. Le marché de la photographie, 1919-1939. Tome 1 ; Les usages de la photographie, 1919-1939. Tome 2, Paris ; Montréal, L'Harmattan, « Champs visuels ».

Deluermoz Q., Fureix E., Charpy M., Joschke C., Le Men S., Mcwilliam N. et Schwartz V. (2014), « Le XIX ${ }^{\mathrm{e}}$ siècle au prisme des visual studies », Revue d'histoire du XIX ${ }^{e}$ siècle, n ${ }^{\circ}$ 49, p. 139-175.

Duboeuf M., Faure G. et Frécon R. (1996), « Le travail ordinaire de trois photographes d'usine », in Peroni M. et Roux J. (dir.), Le Travail photographié, Saint-Étienne, Publications de l'Université de Saint-Étienne ; Paris, CNRS.

Fleig A. (1997), Étant donné l'âge de la lumière II. Naissance de la photographie comme média, Neuchâtel, Ides \& Calendes.

Francastel P. (1956), Art et technique aux XIX et XX siècles, Paris, Denoël, Gonthier, « Bibliothèque Médiations ».

Freund G. (1974), Photographie et société, Paris, Seuil, « Points ».

Fried M. (2013), Pourquoi la photographie a aujourd'hui force d'art?, Malakoff, Hazan.

Gastaut A. (dir.) (2006), La Photographie publicitaire en France: de Man Ray à Jean Goude (catalogue de l'exposition présentée aux Rencontres internationales de photographie d'Arles, 4 juillet-17 septembre 2006 ; Arts décoratifs, Musée de la publicité, Paris, dans le cadre du mois de la photo, 7 novembre 2006-25 mars 2007), Paris, Les Arts décoratifs.

Hoggart R. (2013), « Humanities et culture de masse », in Claustres A. (traduction de Françoise Jaouën), Le Tournant populaire des Cultural Studies : l'histoire de l'art face à une nouvelle cartographie du goût, 1964-2008, Dijon, Presses du réel, « CEuvres en société. Anthologies ».

Josué I. (1996), « De la photo documentaire à la photo publicitaire. Débat avec quatre photographes professionnels », in Peroni M. et Roux J. (dir.), Le Travail photographié, SaintÉtienne, Publications de l'Université de Saint-Étienne ; Paris, CNRS.

Lugon O. (2001), Le Style documentaire : d'August Sander à Walker Evans, 1920-1945, Paris, Macula.

Méaux D. et Dancer-Mourès M. (dir.) (2014), Les Photographes et la commande industrielle. Autour des Éditions Paul-Martial, Saint-Étienne, musée d'art moderne et contemporain.

Peroni M. et Roux J. (dir.) (1996), Le Travail photographié, Saint-Étienne, Publications de l'Université de Saint-Étienne ; Paris, CNRS.

Rivallin M. et Viewing P. (dir.) (2016), François kollar. Un ouvrier du regard (catalogue de l'exposition de la galerie du Jeu de paume de Paris, 9 fevvrier-22 mai 2016 ; Slovak national gallery, Bratislava, 9 juin-11 septembre 2016), Paris, La Martinière ; Jeu de paume.

Schaeffer J.-M. (1996), Les Célibataires de l'art : pour une esthétique sans mythes, Paris, Gallimard, « NRF essais ».

Woronoff Denis (2003), La France industrielle : gens des ateliers et des usines, 1890-1950, Paris, Chêne. 


\section{NOTES}

1. Dès les années 1930, les travaux menés par Gisèle Freund ont renouvelé la manière d'aborder la photographie en tant qu'objet social.

2. L'entreprise répond aux commandes de plusieurs entreprises de métallurgie, du bâtiment et travaux publics, de la construction mécanique, de la fabrication textile, de l'extraction d'énergie et de l'industrie pharmaceutique. Elle compte notamment parmi ses clients la SNCF, les Forges et Aciéries de la Marine et d'Homécourt, Schneider, l'OTUA, les chantiers navals de Penhoët, Citroën...

3. L'entreprise semble avoir débuté une activité photographique propre en 1929, date des premiers négatifs et tirages (cette activité ayant pu jusque-là être sous-traitée). La production photographique de l'entreprise se décline en trois pratiques: la photographie de reportage, réalisée à l'extérieur, qui est désignée par la mention «sortie» au sein des carnets de référencement des négatifs ; la photographie de studio, désignée par « atelier "; la reproduction des maquettes, dessins, photographies, affiches... qui sont parfois réintégrés dans les compositions publicitaires, désignée par « repro » ou « maq. ».

4. Selon les besoins de la publication, l'entreprise missionne ses photographes pour la réalisation de reportages sur le terrain (généralement lorsqu'un grand nombre de clichés doit être réalisé), ou fait appel à des photographes indépendants (pour la réutilisation d'une ou quelques vues). On retrouve parfois les mentions des photographes en fin de publication : lorsqu'il s'agit de clichés réalisés par les photographes de l'entreprise, la mention «Photographies Éditions Paul-Martial » ne précise pas le nom du photographe; les noms des photographes indépendants sont en revanche parfois mentionnés.

5. D’après les carnets de référencement des négatifs, pour partie conservés dans les fonds du MAMC+ et de Marc Pagneux.

6. Vente aux enchères organisée par la maison Acalif à Chartres le dimanche 6 octobre 1991. L'expert en photographie et marchand Marc Pagneux est chargé de l'évaluation d'« un fonds d'archives de l'entreprise des Éditions Paul-Martial de près de 12000 photographies réparties en 94 lots thématiques. 11 autres lots concernent des "maquettes et photos retouchées", des tirages de L. Le Boyer, Pierre Boucher, René Zuber, René-Jacques, Émeric Feher, François Kollar, des revues photographiques Zoom et des brochures et maquettes publicitaires des Éditions PaulMartial ». Source : brochure de la vente aux enchères présente dans le fonds du MAMC+.

7. On trouve le crédit de ces photographes dans certaines publications des Éditions Paul-Martial. Ces opérateurs indépendants fournissent régulièrement leurs épreuves aux agences, studios publicitaires, journaux et éditeurs. Les mentions faites de leurs noms sont généralement rassemblées en de fin de publications et ne permettent pas de révéler la parenté de chacune des photographies.

8. Ces trois photographes retraités, ayant travaillé dans les usines de Creusot-Loire entre 1950 et 1970, comparent leur expérience de photographes d'entreprise: Marcel Duboeuf à l'usine de l'Ondaine à Firminy-Unieux, Gaston Faure à l'usine du Marais de Saint-Étienne, René Frécon à l'usine de Saint-Chamond qui a été photographiée par le photographe des Éditions Paul-Martial, S. Nicolas, au début des années 1930 à l'époque de la FAMH.

9. Le concept de «monde » et, plus précisément, les modalités de l'« être-au-monde », sont au fondement du texte "Être et Temps» de 1927 de Martin Heidegger. Celui-ci considère que le Dasein (l'« être présent ») est continuellement absorbé dans une activité pratique pour laquelle il utilise des objets à des fins utilitaires. 


\section{RÉSUMÉS}

Créée à Paris en 1926, l'entreprise des Éditions Paul-Martial bénéficie d'une renommée importante entre les deux guerres. Elle se spécialise dans l'industrie et propose à ses clients plusieurs types de prestations comme l'édition de catalogues publicitaires dans lesquels la photographie tient une place de choix. De nombreux reportages sont réalisés entre le début des années 1930 et la fin des années 1960 auprès des forges, aciéries, métallurgies, des laboratoires pharmaceutiques, des entreprises agricoles, de mécanique, de matériaux de construction, de fabrication textile et de transport ferroviaire du territoire français. Les archives photographiques de l'entreprise qui sont conservées au musée d'art moderne et contemporain de Saint-Étienne Métropole permettent d'observer les modalités du travail au début du $\mathrm{xx}^{\mathrm{e}}$ siècle et les représentations publicitaires qui les accompagnent. Selon la visée, les vues feignent une captation sur le vif ou assument une théâtralisation de l'activité.

Created in Paris in 1926, the Éditions Paul-Martial benefits from an important reputation between the two world wars. The Éditions Paul-Martial was specialized in the field of the industry and offered several kinds of services such as the publication of advertising catalogues in which photography plays a key role. Many reports were produced between the early 1930's and the end of the 1960's by forges, steel mills, metallurgy, pharmaceutical laboratories, agricultural companies, mechanical engineering, construction materials, textile manufacturing and rail transport companies in France. The archives of the company's photographic studio, which are kept at the Musée d'art moderne et contemporain de Saint-Étienne Métropole, allow us to observe the working methods of the beginning of the 20th century and the advertising representations accompanying it. Depending on the aim, the views pretend to be a live capture or assume a staged of the activity.

\section{INDEX}

Mots-clés : photographie, édition publicitaire, industrie, homme au travail

Keywords : photography, advertising publishing, industry, man at work

\section{AUTEUR}

\section{ANNE-CÉLINE CALLENS}

Anne-Céline Callens est Maître de conférences en Sciences de l'art à l'Université Jean Monnet de Saint-Étienne, membre du Centre Interdisciplinaire d'Études et de Recherche sur l'Expression Contemporaine (CIEREC - EA 3068). Elle a été chercheur invité à la Bibliothèque nationale de France en 2014/2015 (département des estampes et de la photographie) et attachée de conservation du patrimoine, chargée de mission scientifique au Musée d'art moderne et contemporain de Saint-Étienne Métropole (MAMC+) en 2017. Elle a été co-commissaire de l'exposition Les Éditions Paul Martial : la belle publicité (avec Martine Mourès-Dancer, MAMC+, 2014) et commissaire des expositions Regard(s) sur l'industrie (Médiathèque de Saint-Étienne, 2015) et Coup de Pub : graphisme et publicité en France dans les années 1930 (MAMC+, 2019). Elle a coordonné les ouvrages Art, architecture, paysage. A l'époque post-industrielle (avec Pauline Jurado-Barroso, PUSE, 2015) et Photographier le chantier : transformation, inachèvement, altération (avec Jordi Ballesta, Hermann, 2019). 